\title{
IMPACT DES MESURES SATELLITALES SUR LA PRÉVISION NUMÉRIQUE
}

\author{
Jean Pailleux \\ Météo-France \\ Centre national de recherches météorologiques \\ 42, avenue Gaspard-Coriolis \\ 31057 Toulouse Cedex
}

RÉSUMÉ Depuis une vingtaine d'années, les modèles de prévision numérique utilisent quotidiennement certaines données des satellites météorologiques. Après une présentation sommaire des instruments et des traitements à l'origine de ces données, cet article essaie de chiffrer leur impact sur la qualité de la prévision numérique. Pour cela, on dresse un historique de l'utilisation des données satellitales dans les modèles de prévision numérique, depuis la fin des années soixante-dix, et on s'appuie sur plusieurs études d'impact effectuées dans quelques grands centres de prévision numérique, en particulier au Centre européen pour les prévisions météorologiques à moyen terme, se trouvant à Reading en Angleterre.

\begin{abstract}
Impact of satellite measurements on numerical forecasting
For about twenty years, numerical prediction models have used satellite data on a daily basis. After a brief description of the instruments and the processing of the data, this paper tries to quantify their impact on the quality of the numerical forecasts. A review of the use of satellite data in numerical weather prediction since the end of the seventies is given, using the results from several impact-studies carried out in a few important centres, especially at the European Centre for Medium range Weather Forecasts, situated in Reading, England.
\end{abstract}

\section{LES SATELLITES EN PRÉVISION NUMÉRIQUE, UNE HISTOIRE DÉJÀ ANCIENNE !}

Ceux, nombreux, qui pensent que la prévision numérique n'utilise les données de satellites que depuis une date récente se trompent ! C'est en effet vers les années 1975-1976 que les analyses objectives françaises commencent à utiliser les premiers profils de température et les premières estimations de vent obtenus à partir de satellites. Les profils verticaux sont des sondages infrarouges de température fabriqués par les Américains à partir de l'instrument $\mathrm{VTPR}^{(1)}$ embarqué sur les satellites NOAA à orbite polaire. Les vents de satellite sont obtenus à partir des premiers satellites géostationnaires américains Goes, par observation du déplacement des nuages.

Cette utilisation française des données issues de satellites n'a rien d'exceptionnel : dans le monde, les centres de prévision numérique analogues au centre français commencent à utiliser profils et vents de satellite à peu près à la même époque. Jusque vers la fin des années soixante-dix, la plupart des modèles opérationnels sont limités à une partie de l'hémisphère nord ; il n'y a pas de modèle opérationnel couvrant la totalité du globe terrestre et seul le service météorologique australien utilise en routine un modèle couvrant l'hémisphère sud. L'information satellitale est vue par l'usager modélisateur comme une information d'appoint, importante pour compléter les réseaux conventionnels d'observation sur les océans de l'hémisphère nord (sauf bien sûr pour les Australiens qui souffrent d'un manque criant d'observations classiques sur leur hémisphère). Peu de modélistes se préoccupent à l'époque d'améliorer les algorithmes pour utiliser ces données et d'évaluer précisément leur impact. 
Un événement, important dans le monde météorologique et préparé de longue date, a lieu de décembre 1978 à novembre 1979 : il s'agit de l'expérience mondiale du Garp (Global Atmospheric Research Program). Cette expérience consiste, pour les nombreux pays participants, à faire un effort sans précédent, pendant un an, pour observer au mieux l'atmosphère en globalité. De nombreux systèmes d'observation spéciaux sont mis en place (« dropsondes », ballons à niveau constant, bouées dérivantes) et, en plusieurs régions du globe, les réseaux d'observation existants sont renforcés.

C'est à l'occasion de l'expérience mondiale du Garp que sont mis en place les principaux systèmes d'observation par satellite qui sont encore opérationnels aujourd'hui, en particulier pour la prévision numérique. Décrivons brièvement ces systèmes.

\section{Le Tovs sur les satellites à orbite polaire NOAA}

Le premier instrument Tovs est mis en orbite en 1978, juste avant l'expérience mondiale du Garp, sur le satellite américain Tiros $N$, qui est le prototype d'une série prolongée par neuf autres satellites, de NOAA 6 à NOAA 14, le lancement de ce dernier ayant lieu le 30 décembre 1994. Malgré l'échec de la mission NOAA 13 et une panne d'instrument qui affecte $N O A A 8$, la NOAA réussit à fournir aux usagers météorologistes des données Tovs en provenance de deux satellites à orbite polaire à chaque instant (l'un en quadrature par rapport à l'autre), et cela presque sans interruption depuis 1979. Dire que les deux satellites sont en quadrature signifie qu'à chaque instant les plans de leurs orbites sont perpendiculaires, ce qui optimise la couverture spatiales des données (figure 1). Le Tovs est en fait un ensemble de trois sondeurs :

- le HIRS 2 (High Resolution Infrared Sounder) qui mesure des radiances (ou luminances énergétiques spectrales) dans vingt canaux infrarouges ;

- le MSU (Microwave Sounding Unit) qui mesure des radiances dans quatre canaux micro-ondes ;

- le SSU (Stratospheric Sounding Unit) qui échantillonne la haute stratosphère dans trois canaux micro-ondes ; un satellite NOAA sur deux seulement est équipé du SSU.

Une description détaillée de l'instrument Tovs se trouve dans Smith et al. (1979). Les radiances Tovs mesurées apportent des informations sur la température et l'humidité dans l'atmosphère, auxquelles elles sont reliées par l'équation de transfert radiatif.

\section{Les sondages atmosphériques Satem}

Un radiomètre embarqué sur un satellite mesure, dans une (ou plusieurs) bande(s) spectrale(s) sélectionnée(s), la puissance du rayonnement provenant de la zone de visée. À partir de cette puissance (en watts), on déduit la radiance (en watts $/ \mathrm{m}^{2}$ ). Connaissant le profil de la composition chimique de l'atmosphère dans la zone de visée, notamment les teneurs en gaz carbonique, ozone et vapeur d'eau qui sont les principaux absorbants, ainsi que la répartition verticale des aérosols et des gouttelettes d'eau, l'équation de transfert radiatif permet de calculer cette radiance. Ce calcul décrit les phénomènes de diffusion et d'absorption par la matière atmosphérique ainsi que son émission propre.

Mais on s'intéresse au problème inverse : à partir des radiances mesurées dans différentes bandes spectrales, il s'agit de faire un sondage de l'atmosphère, c'està-dire, dans le cas du Satem, de restituer les profils de température et d'humidité. Ce problème inverse est plus difficile à résoudre que le problème direct car la solution n'est pas unique ; compte tenu du nombre limité de bandes spectrales utilisées et des imperfections de la mesure, il existe même une infinité de profils compatibles avec les radiances mesurées. Aussi, les calculs sont-ils effectués de façon itérative à partir d'une ébauche de profil. Dans tous les cas, la qualité du profil restitué est meilleure en l'absence de nuages.

Le sondeur HIRS 2 de l'instrument Tovs utilise vingt canaux et sa résolution horizontale est de $42 \mathrm{~km}$. À titre de comparaison, l'imageur AVHRR utilise quatre ou cinq canaux pour une résolution horizontale de $1,1 \mathrm{~km}$. Pour le sondage de température, le HIRS 2 combine l'information de deux bandes spectrales d'absorption du dioxyde de carbone : sept canaux dans la bande $15 \mu \mathrm{m}$ et six canaux dans la bande 4,3 $\mathrm{mm}$. L'humidité est mesurée à l'aide de trois canaux dans la bande d'absorption de la vapeur d'eau à 6,3 $\mu \mathrm{m}$. Le canal à 9,7 $\mu \mathrm{m}$ est utilisé pour les mesures d'ozone. Trois canaux sont situés dans la fenêtre atmosphérique : les canaux à $11,11 \mu \mathrm{m}$ et $3,76 \mu \mathrm{m}$ pour la détermination de la température de surface et le canal à 0,69 $\mu \mathrm{m}$ pour détecter les nuages.

Informations tirées du livre de Kidder et Vonder Haar (1996). 


\section{Distribution typique d'observations satellitales}
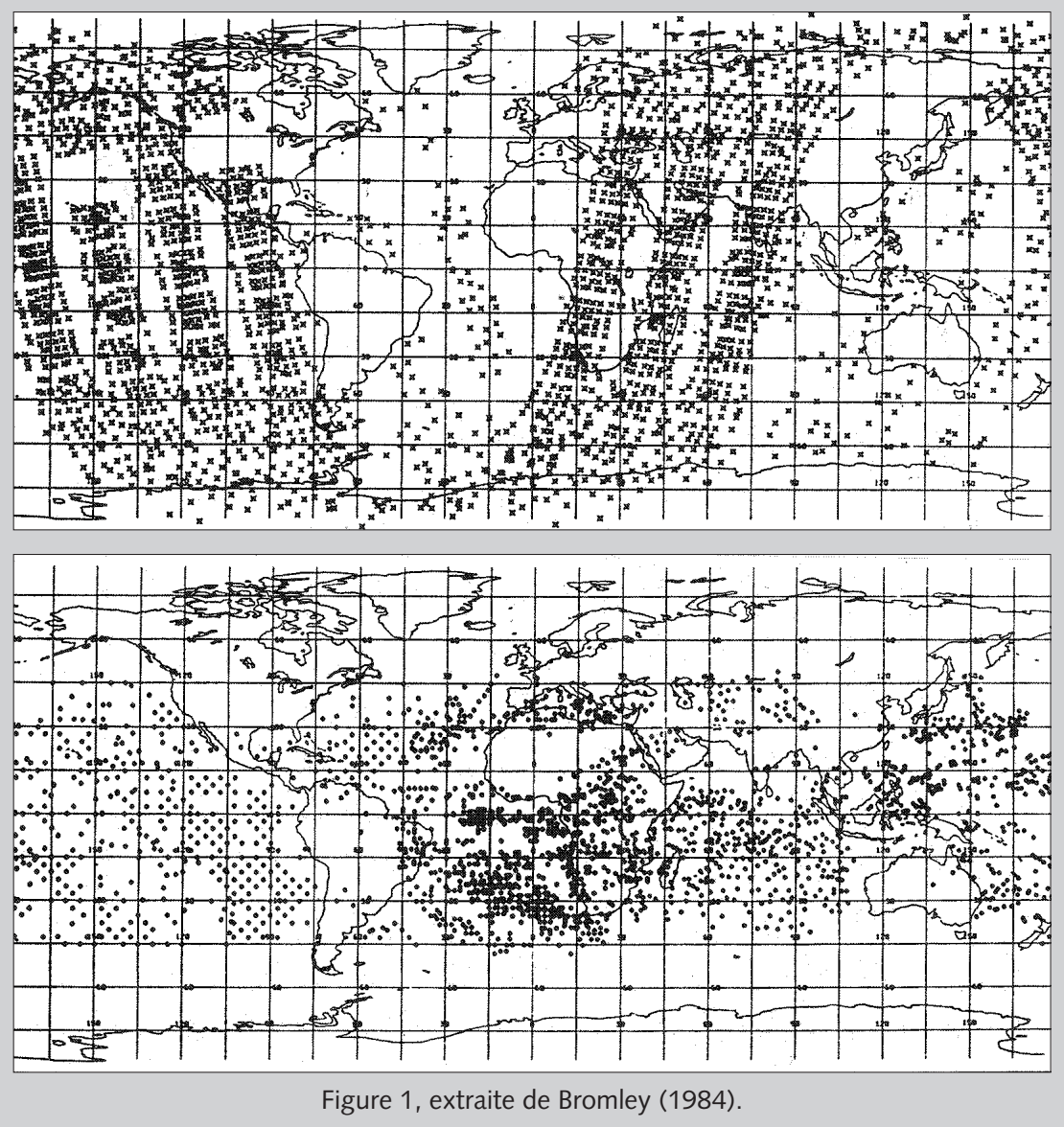

Ces deux cartes montrent la distribution sur le globe des données Tovs (en haut) et Satob (en bas) pour une période de six heures centrée sur le 27 février 1979, à 0 h UTC. Ces données sont typiques de ce qui était disponible pendant l'expérience mondiale du Garp de 1978-1979, mais elles reflètent aussi assez bien la réalité opérationnelle en 1995 !... Notons toutefois quelques détails :

- En février 1979, il n'y avait encore qu'un satellite polaire équipé de l'instrument Tovs (le satellite Tiros N); depuis l'automne 1979 et le lancement de NOAA 6, il y a eu presque en permanence deux satellites en quadrature, ce qui donne une couverture complète du globe sur chaque période de six heures.

- Les Satem distribués sur le Système mondial de télécommunications correspondent à une densité horizontale de l'ordre de 500 km, c'est-à-dire environ deux fois plus faible que ce que l'on voit sur les traces orbitales de la figure du haut.

- La couverture en données Satob (vents de satellite) correspond à trois satellites Goes (dont un à la longitude de l'océan Indien), un satellite Météosat et un satellite japonais GMS. Ce dispositif a été mis en place à l'occasion de l'expérience mondiale du Garp. II a légèrement changé par la suite. En 1996, des Satob sont produits à partir de deux satellites Goes (Pacifique, Amérique, Atlantique), d'un Météosat (Atlantique, Europe, Afrique), d'un satellite indien (océan Indien, Asie, production très limitée) et d'un satellite japonais (Ouest Pacifique, Australie). Pour plus de détails sur les vents Satob et leur production opérationnelle, voir Nuret (1990).

À partir de ces différents jeux de radiances, le service américain Nesdis s'efforce de restituer des profils de température et d'humidité sur tout le globe, en temps réel, par une inversion de l'équation de transfert radiatif. Ce même service distribue sur le Système mondial de télécommunications (SMT) de la Veille météorologique mondiale un échantillon de profils restitués, avec une résolution horizontale d'environ $500 \mathrm{~km}$. Ces sondages sont généralement appelés Satem (du nom du code OMM conçu spécialement pour les transmettre sur le SMT vers la fin des années soixantedix), ou encore « Satem $500 \mathrm{~km}$ », pour les distinguer d'autres jeux de données Tovs, à plus haute résolution spatiale, également distribués par le Nesdis à plusieurs centres météorologiques. 
Les satellites géostationnaires et les Satob
C'est également juste avant l'expérience mondiale de 1978-1979 qu'est lancé le premier satellite Météosat, stationnaire sur l'équateur vers $0^{\circ}$ de longitude. Avec le lancement de nouveaux satellites du même type (américains et japonais), l'on peut obtenir ainsi, pour la première fois en décembre 1977, une couverture de toute la ceinture équatoriale par les instruments des satellites géostationnaires. Ces satellites sont équipés de radiomètres observant la Terre dans l'infrarouge, le visible, ainsi que d'un canal infrarouge sensible à la vapeur d'eau dans le cas de Météosat. Contrairement aux satellites à orbite polaire, l'observation peut se faire de manière très fréquente dans le temps : une image de la Terre et de sa couverture nuageuse est prise généralement toutes les trente minutes. Cet ensemble d'images permet de suivre le déplacement de certains nuages, d'où l'on déduit une mesure de vent. Ces mesures de vent sont connues depuis cette époque sous le nom de Satob (code OMM conçu spécialement pour transmettre les données de satellites en même temps que le code Satem); elles sont du même type que les vents des satellites Goes déjà utilisés quelques années auparavant. Le lecteur assidu de La Météorologie est déjà très familiarisé avec la technique de production des vents Satob, puisqu'il a pu lire l'article de Nuret (1990).

Satem et Satob constituent depuis 1979 les deux systèmes d'observation satellitale employés couramment en prévision numérique. Les deux cartes de la figure 1 montrent une couverture typique du globe en données Satem et Satob disponibles sur une tranche horaire de six heures. Signalons toutefois un autre système d'observation, apparu en 1991 avec le lancement du satellite européen ERS 1. Ce satellite est équipé de divers instruments, dont un diffusiomètre qui mesure des sections efficaces de rétrodiffusion à la surface de l'océan, permettant d'obtenir des informations sur le vent en surface. Au début de 1995, ces données de diffusiomètre n'étaient utilisées dans les modèles opérationnels qu'en GrandeBretagne. Nous ne décrivons pas leur impact dans cet article car, sur ce sujet, les expériences numériques sont trop rares et le recul insuffisant.

Le $1^{\text {er }}$ août 1979, avec les débuts opérationnels à Reading du Centre européen pour les prévisions météorologiques à moyen terme (CEPMMT), commence la première utilisation quotidienne d'un modèle «global » (couvrant la totalité du globe terrestre). En conséquence, il convient d'accorder une plus grande attention à la qualité de l'analyse sur tout le globe et aux systèmes d'observation par satellite, susceptibles de jouer un rôle déterminant sur l'hémisphère sud, sous les tropiques et, peut-être, sur les océans de l'hémisphère nord. Par ailleurs, le CEPMMT est chargé de produire en différé des analyses globales pour toute l'année de l'expérience mondiale du Garp, à l'aide de son système opérationnel d'assimilation de données. Les modélistes commencent alors à s'intéresser sérieusement à l'impact des observations satellitales sur la qualité de la prévision numérique, d'autant que les scientifiques qui ont développé les nouveaux instruments sont curieux de connaître cet impact. L'évolution de beaucoup de centres de prévision numérique vers l'emploi des modèles globaux va s'accentuer au cours des années quatre-vingt, stimulant l'intérêt opérationnel pour les données de satellite.

Les premières expériences importantes destinées à évaluer l'impact des données satellitales sur la prévision numérique sont donc faites au début des années quatre-vingt, sur des périodes de l'expérience mondiale du Garp, surtout à l'initiative du CEPMMT à Reading et de quelques équipes américaines. L'étude de Kållberg et al. (1982) conclut que les Satob ont un rôle important pour l'analyse du vent et la prévision à court terme sous les tropiques, un impact positif modéré sous les latitudes moyennes de l'hémisphère sud, et pas d'impact vraiment discernable aux latitudes moyennes de l'hémisphère nord. L'article de Halem et al. (1982) conclut que les Satem ont un rôle décisif pour définir la structure tridimensionnelle de grande échelle de l'atmosphère dans une analyse.

L'impact notable des Satem dans l'hémisphère sud et des Satob sous les tropiques, sur les analyses et les prévisions numériques, se trouve confirmé dans toutes les études d'impact ultérieures, en particulier dans celle de Uppala et al. (1984). Ces derniers s'attachent cependant en particulier à étudier l'impact de ces systèmes d'observation sur l'hémisphère nord, et montrent (figure 2) l'importance du choix de la période et du type de circulation atmosphérique. En fait, ils observent que l'impact des données satellitales (Satem plus Satob) sur l'hémisphère nord peut être nettement positif à certains moments (comme la période de novembre 1979 qu'ils étudient) et nul à d'autres moments (comme en février 
1979). Cela indique qu'il existe vraisemblablement, suivant la région et suivant la situation météorologique, de fortes variations de la sensibilité d'un modèle aux conditions initiales.

\section{Impact comparé sur l'hémisphère nord des données conventionnelles et satellitales}

Uppala et al. (1984) ont comparé trois jeux de prévisions faites avec le modèle du CEPMMT au début des années quatre-vingt :

- Le premier contient tous les types d'observations disponibles. Noté « Al » (All Included), il correspond aux courbes en tireté.

- Le deuxième contient seulement les observations conventionnelles, radiosondes (Temp et Pilot) et données de surface (Synop, Ship et bouées). Noté "SO » (Surface-based Only), il correspond aux courbes en trait plein.

- Le troisième contient les observations spatiales plus celles qui peuvent être transmises par des satellites (observations de pression en surface, observations d'avion automatisables). Noté "SP » (Space-based), il correspond aux courbes en pointillé.

Chaque diagramme indique (en ordonnée) les scores moyens des trois jeux de prévision sur une période donnée en fonction de l'échéance de la prévision (jusqu'à dix jours). Le diagramme du haut correspond à sept prévisions d'une période de novembre 1979, celui du bas à neuf prévisions d'une période de février 1979. Le score choisi est la corrélation d'anomalie du géopotentiel moyennée de 1000 à $200 \mathrm{hPa}$; la prévision est d'autant meilleure que la corrélation est proche de $100 \%$. On considère aussi qu'en dessous de $60 \%$ une prévision est trop mauvaise pour être utilisable.

Notons que, dans la première période (novembre 1979), la prévision contenant toutes les observations est bien meilleure que chacune des deux autres, ce qui montre que systèmes conventionnels et satellitaux se complètent bien.
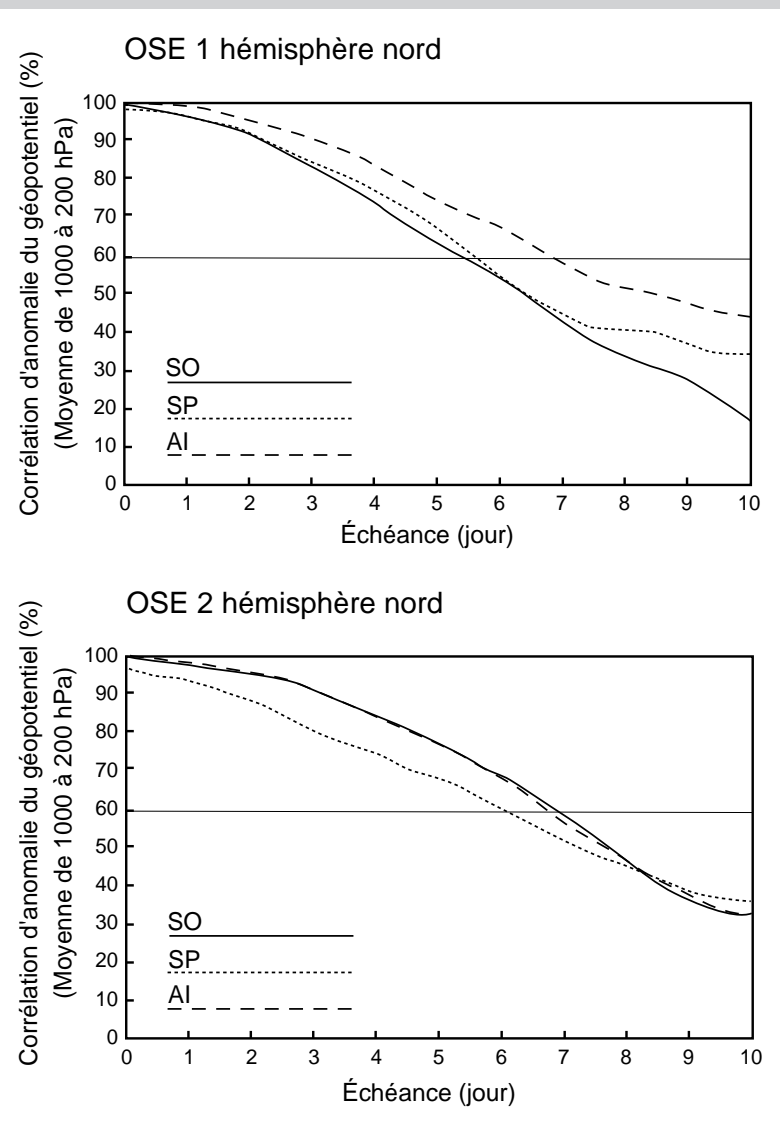

Inversement dans la deuxième période, la prévision ne contenant pas les données satellitales (SO) est aussi bonne que celle contenant toutes les données. Nous obtenons donc un fort impact positif des systèmes satellitaux sur une période, pas sur l'autre. Par ailleurs, Uppala et al. (1984) ont aussi évalué l'impact séparé des Satem et des Satob. Ils confirment le même résultat pour l'impact des Satem seuls.

Figure 2, extraite de Uppala et al. (1984). 
L'ASSIMILATION DES DONNÉES SATELLITALES
L'expérience mondiale du Garp, l'arrivée du Tovs et les débuts opérationnels du CEPMMT ont coïncidé avec une généralisation des méthodes d'analyse par interpolation optimale multivariée dans les centres de prévision numérique. Le lecteur peut se reporter à Pailleux (1992) pour une description de l'algorithme d'interpolation optimale et de la façon dont il traite les données issues de satellites. Rappelons simplement que le schéma standard opérationnel consiste à corriger toutes les six heures $(0 \mathrm{~h}, 6 \mathrm{~h}, 12 \mathrm{~h}$ et $18 \mathrm{~h}$ UTC) la prévision numérique fournie par le modèle (ébauche ou « guess ») par une interpolation spatiale des écarts au modèle constatés aux points d'observation. Il s'agit donc d'une assimilation intermittente des observations (toutes les six heures) plutôt que continue dans le temps.

L'interpolation spatiale est de nature statistique ; elle fait intervenir la structure statistique des erreurs de l'ébauche et des observations. Dans l'assimilation, la pondération de chaque variable observée tient compte d'un écart type d'erreur d'observation (qui chiffre le degré de confiance à attribuer à l'observation) et de la distribution géographique de l'ensemble des observations sur la zone à analyser. L'analyse est «multivariée » en ce qui concerne le vent, le géopotentiel et la température, c'est-à-dire que toutes ces variables sont analysées simultanément, une observation de vent apportant aussi des informations sur le géopotentiel et la température, et réciproquement. L'analyse d'humidité est plutôt traitée à part, au moyen d'une interpolation optimale univariée.

Les observations Satem entrent sous forme d'épaisseurs géopotentielles dans une interpolation optimale multivariée. Les liens statistiques qui relient vent, température et géopotentiel s'étendent facilement à une épaisseur géopotentielle qui représente une température moyenne. Les humidités Satem trouvent aussi facilement leur place dans une analyse d'humidité sous forme d'humidité moyenne de certaines couches. Quant aux vents Satob, ils sont traités comme toute observation de vent in situ.

Remarquons surtout que, dans ce type d'algorithme, le traitement des données satellitales n'est pas fondamentalement différent de celui des données conventionnelles. On assimile les données sous forme de données inversées ; rien n'est fait pour prendre en compte les problèmes spécifiques des mesures de radiance qui constituent pourtant l'information de base. On va voir que cela est une faiblesse sérieuse des schémas d'assimilation de données, faiblesse qui apparaît clairement lorsque l'on étudie en détail l'impact des données satellitales sur les analyses et les prévisions numériques.

Au cours de la décennie quatre-vingt, les études d'impact des Satem et des

\section{LES PÉRIODES} DE DOUTE

Satob se multiplient. Les résultats sont toujours cohérents pour montrer un fort effet bénéfique des Satem dans l'hémisphère sud et des Satob sous les tropiques. Mais l'impact dans l'hémisphère nord des Satem devient, dans beaucoup d'expériences numériques, négligeable en moyenne, avec autant de cas d'impact positif que d'impact négatif ! Gallimore et Johnson (1986) signalent que l'utilisation de données Tovs peut dégrader la façon dont est rendue la structure thermique de certains systèmes dépressionnaires. Kelly et Pailleux (1988) obtiennent un impact globalement neutre des Satem sur l'hémisphère nord en comparant deux semaines de prévision, avec et sans usage de ces données, dans le système opérationnel du CEPMMT. Une étude analogue décrite par Andersson et al. (1991) aboutit même à un impact légèrement négatif en moyenne. L'examen détaillé des cas d'impact négatif, documenté par Kelly et al. (1991), attire l'attention sur certains problèmes d'inversion du Tovs, aboutissant, dans la mise en œuvre du Nesdis, à des profils de température de qualité très médiocre dans certaines masses d'air. Ces profils, mal filtrés ou mal pondérés par le schéma d'analyse (dans ce cas le schéma opérationnel au CEPMMT), dégradent localement de façon assez sérieuse l'analyse et la prévision opérationnelles. Un aperçu des problèmes évoqués par Andersson et al. (1991) et par Kelly et al. (1991) est donné dans la figure 3 et ses commentaires.

Au début des années quatre-vingt-dix, il se dit (dans tous les milieux «bien informés ») que les données de satellite ne servent à rien sur l'hémisphère nord en prévision numérique. Cette impression vient des expériences précitées sur les données Tovs ; elle est confirmée par les études récentes sur les Satob (Radford, 1989 ; Kelly et Pailleux, 1989) montrant que la détermintation du vent à partir de 
nuages rencontre, elle aussi, des problèmes difficiles à résoudre. Ces problèmes entraînent parfois une forte sous-estimation de la vitesse du vent Satob dans les jets, ainsi que des cas d'impact négatif sur la prévision. Enfin, cette impression n'est pas vraiment remise en cause par les modèles opérationnels à résolution fine et à domaine limité, qui incorporent pourtant souvent l'information satellitale de manière un peu différente. Parmi eux, citons :

- le modèle Péridot, opérationnel en France de 1985 à 1993, qui utilise dans son analyse les radiances Tovs (associées à des informations de l'imageur AVHRR sur les nuages) fournies par le Centre de météorologie spatiale de Lannion et assimilées directement (Durand, 1985);

\section{Comment l'impact des Satem peut être négatif}

Kelly et al. (1991) et Andersson et al. (1991) ont identifié sur le cas du 12 janvier 1989, à 12 h UTC, de fortes erreurs sur les profils de température Satem inversés par le service américain Nesdis. La carte a représente le champ de température virtuelle moyenne de la couche 1 000-700 hPa extrait de l'ébauche du CEPMMT. À chaque point d'observation Satem, est pointé (en degrés K) l'écart entre l'observation Satem et l'ébauche. La carte b et identique à a mais pour la couche 500-300 hPa. Notons les forts écarts positifs au sud de Terre-Neuve dans la couche $1000-700 \mathrm{hPa}$, là où de l'air froid continental a pénétré au-dessus de l'océan. II est clair que le Tovs ne réussit pas à localiser l'air froid de basse couche, au-dessus d'un océan beaucoup plus chaud; cette erreur se trouve compensée par une erreur de signe opposé dans la haute troposphère, comme l'indiquent les écarts négatifs pour la couche 500$300 \mathrm{hPa}$. Notons aussi un problème analogue au milieu de I'Atlantique, dans l'air chaud de l'ondulation (mais avec un signe opposé). Dans tous ces cas, le gradient vertical de température entre la haute et la basse trotosphère est vraiment très faux.

Le diagramme de dispersion c montre la comparaison des scores de 15 prévisions (en termes de corrélation d'anomalie) faites avec et sans les données Satem. Un impact des Satem sur une prévision se traduit par un point au-dessus de la diagonale. Comme en moyenne la position du point est plutôt au-dessous de la diagonale ( $x$ $x$ indique le point moyen) et que l'on a plus de points au-dessous qu'au-dessus, I'impact des Satem est légèrement négatif dans cette étude qui couvre une période de février 1987. Les assimilations et les prévisions sont obtenues avec le système opérationnel en juillet $1988 \mathrm{au}$ CEPMMT. Dans les deux publications citées, l'impact négatif des Satem est clairement relié à des problèmes de qualité du type de ceux illustrés ici.

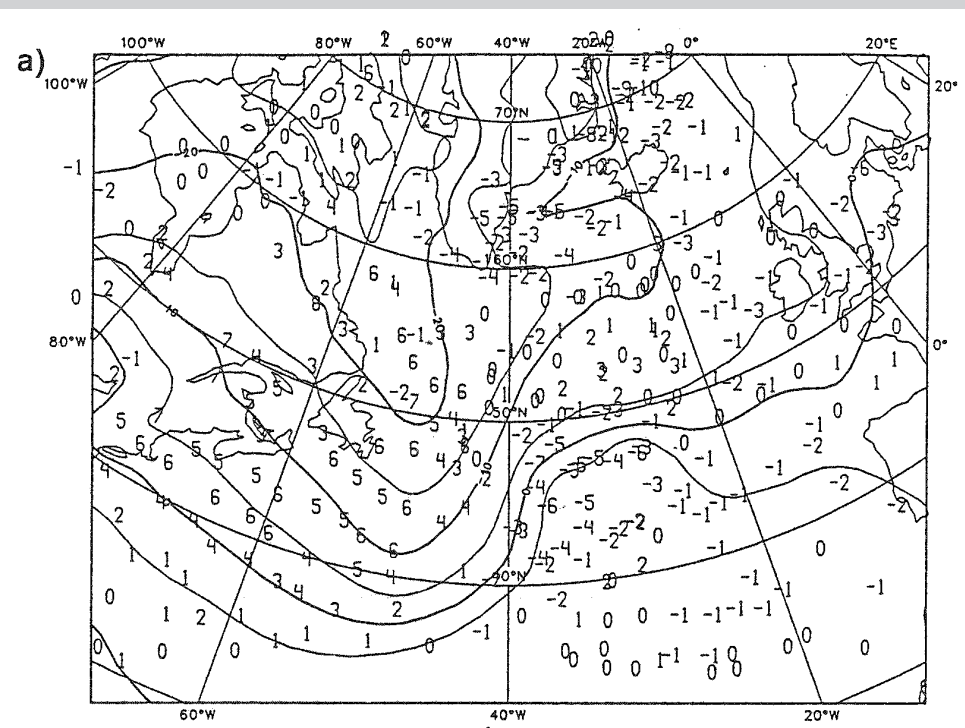

b)

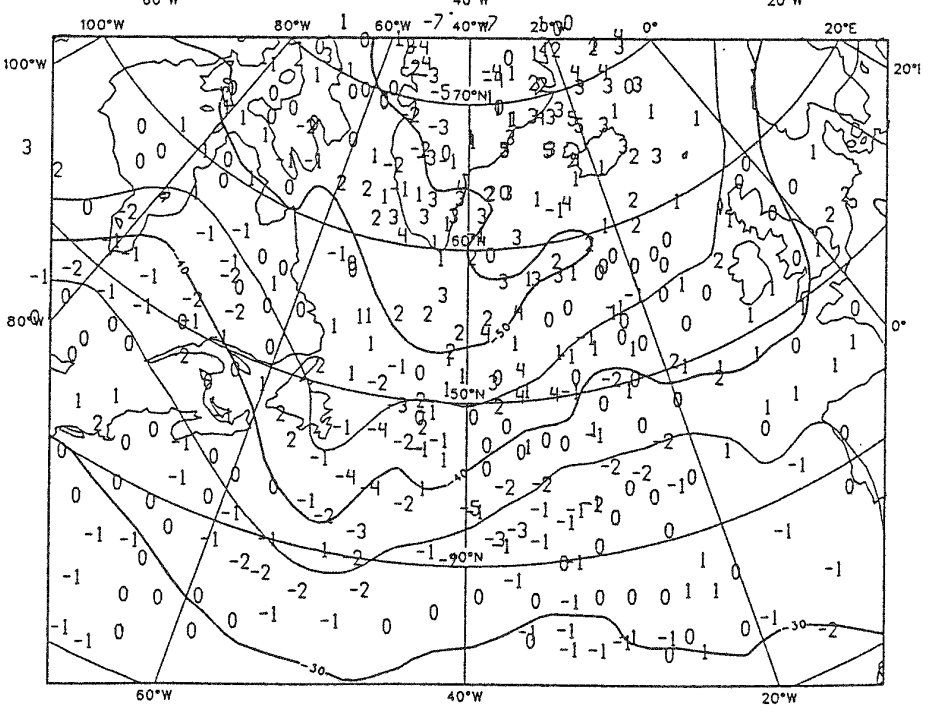

c)

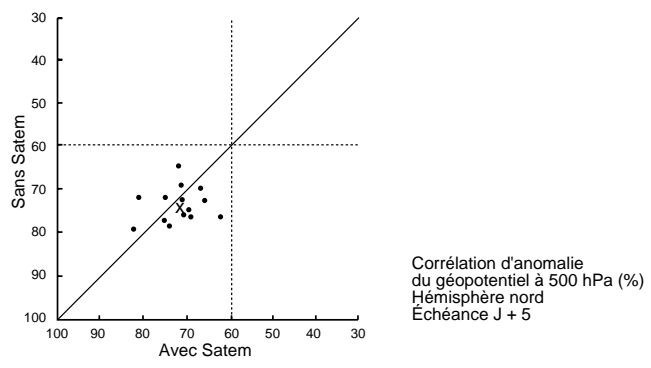

Figure 3, extraite de Andersson et al. (1991) et de Kelly et al. (1991). 
- le modèle anglais à domaine limité, qui utilise pendant plusieurs années des profils Tovs restitués à partir des données reçues au Centre de météorologie spatiale anglais de Lasham (Eyre, 1989 ; Gadd, 1993).

Pourquoi l'impact des données Tovs sur la prévision dans l'hémisphère nord, significatif au début des années quatre-vingt, est-il devenu indétectable une dizaine d'années plus tard? Sans avoir aucune certitude, on peut raisonnablement avancer l'explication suivante : les modèles numériques ont beaucoup progressé en dix ans sur tous les plans (en particulier en résolution), la qualité de beaucoup de stations de radiosondage s'est également améliorée (comme le montrent des statistiques récentes effectuées au CEPMMT). Dans le même temps, l'instrument Tovs est resté le même ; en termes d'impact, le facteur important est la quantité d'information apportée par le Tovs relativement aux autres sources d'information que sont par exemple l'ébauche (donc le modèle lui-même) et les radiosondes. Logiquement, cette part d'information venant de Tovs a dû diminuer depuis 1978. Ainsi, la résolution verticale des modèles opérationnels a pratiquement doublé en dix ans, alors que celle du Tovs (dictée par les caractéristiques de ses canaux) ne donne qu'une information grossière sur le profil vertical de température et d'humidité. De plus, même si les techniques d'inversion du Tovs ont été améliorées, les données Satem restent affectées d'erreurs dont la structure spatiale est complexe et difficile à prendre en compte dans l'analyse. Plus les modèles de prévision numérique ont progressé, plus ils sont devenus sensibles aux conditions initiales, et donc plus ils sont perturbés par des données de qualité médiocre. L'impact du Tovs, mesuré par des expériences numériques, est toujours un bilan entre :

- l'information correcte, non redondante avec les autres systèmes d'observation, apportée par le Tovs, d'une part ;

- la dégradation de l'analyse due aux données Tovs mal inversées et néanmoins acceptées par les divers contrôles de qualité, d'autre part.

Ces dernières, même si elles sont en proportion très faible, peuvent affecter de façon importante la prévision numérique, surtout si elles coïncident avec les zones sensibles de l'analyse. Il est probable que, les modèles devenant de plus en plus sensibles aux mauvaises données au cours des années quatre-vingt, les algorithmes de contrôle de qualité progressant plus lentement, l'aspect « dégradation » a fini par devenir aussi important que l'aspect « amélioration par information correcte » dans le bilan ci-dessus sur l'hémisphère nord. À l'opposé, sur l'hémisphère sud, une information Tovs, même de qualité médiocre, est souvent précieuse compte tenu de l'absence d'autres sources d'information.

VERS UN MEILLEUR USAGE DES MESURES SATELLITALES
Les études d'impact citées plus haut sont généralement effectuées par des chercheurs en prévision numérique soucieux, en premier lieu, d'améliorer l'assimilation des données de satellite, la mesure de l'impact n'étant pas la seule fin en soi. Dès le début des années quatre-vingt, alors que l'impact décevant sur l'hémisphère nord n'alimente pas encore les conversations des comités scientifiques, ces chercheurs commencent à identifier plusieurs faiblesses dans les schémas d'assimilation de données, faiblesses que l'on peut maintenant résumer ainsi :

- L'emploi dans l'analyse des Satem sous forme d'épaisseurs ne permet pas d'extraire l'information réelle contenue dans les radiances satellitales. Un Satem est un profil inversé à partir des radiances Tovs, mais aussi à partir d'un profil initial provenant, soit d'une climatologie élaborée, soit d'une identification de la masse d'air au point considéré. Dans le processus d'inversion, cette information annexe n'est pas du tout négligeable devant l'information « radiances ». Il arrive même, dans certaines masses d'air, qu'elle soit prédominante. Dans certains cas de phénomènes très anormaux en température, cette information annexe peut être complètement erronée et guider l'algorithme d'inversion vers une solution fausse, bien que compatible avec les radiances observées.

- Les structures d'erreur qui en résultent pour les Satem peuvent être très particulières (figure 3) ; elles peuvent même être très éloignées de structures gaussiennes (si par exemple la production de Satem a été affectée par des erreurs sur la détection de nuages), alors que les contrôles de qualité effectués dans l'assimilation de données supposent généralement que ces erreurs sont gaussiennes. 
- Même si un raisonnement strictement identique ne peut être tenu pour les Satob, les erreurs Satob sont aussi assez particulières, et certaines proviennent du fait que ce que l'on traite comme une donnée de vent n'en est pas une : c'est en fait le déplacement d'une forme nuageuse (ou d'une forme observée dans le canal vapeur d'eau).

Il devient donc clair que les algorithmes standard d'utilisation des données satellitales décrits plus haut sont loin d'être optimaux et que leur amélioration passe par une plus grande intégration des processus d'inversion et d'assimilation de données. Plusieurs solutions sont envisagées :

- Idéalement, l'assimilation devrait prendre en compte l'information réelle, telle qu'elle provient de l'instrument du satellite ; dans le cas du Tovs, on devrait donc assimiler directement les radiances correspondant à la vingtaine de canaux de l'instrument. Notons que les travaux de Durand (1985) mis en œuvre dans l'analyse française du modèle Péridot constituent la première (et sans doute encore la seule jusqu'en 1995) tentative opérationnelle réussie. L'analyse Péridot étant fondée sur une interpolation optimale multivariée, on est forcé dans ce contexte d'imposer un lien statistique (donc linéaire) entre corrections en radiance et corrections en température. Cette hypothèse un peu grossière s'est révélée suffisamment robuste et raisonnable pour fonctionner opérationnellement pendant huit ans à Météo-France. Mais elle n'est pas facilement généralisable à toute masse d'air, ce qui explique qu'elle n'ait pas été tentée dans des analyses couvrant une grande portion du globe.

- À défaut, il faudrait assurer une meilleure communication entre les algorithmes d'inversion et les algorithmes d'assimilation de données. Cela suppose par exemple que, pour les produits globaux actuels, le Nesdis (Washington) distribue beaucoup plus de choses que les simples produits inversés.

Les travaux de Le Dimet et Talagrand (1986) et ceux de Lewis et Derber (1985) montrent la puissance des algorithmes variationnels utilisant la «méthode adjointe » pour l'assimilation des données. Courtier et Talagrand (1987) expérimentent la méthode sur des observations réelles à l'aide d'un modèle simple, ce qui en démontre un peu plus l'intérêt opérationnel. Les méthodes variationnelles présentent de nombreux avantages sur les méthodes d'interpolation optimale. Ces avantages sont expliqués plus en détail dans Pailleux (1993), par exemple. Parmi eux, l'un s'avère déterminant pour l'usage des données satellitales : il s'agit de la possibilité, dans les algorithmes variationnels, de prendre en compte toute variable observée, aussi différente soit-elle des paramètres météorologiques du modèle, pour peu que l'on sache la relier à ces paramètres par un opérateur suffisamment régulier. Un algorithme variationnel peut donc assimiler directement une radiance à l'aide du modèle de transfert radiatif approprié et de son adjoint. Cet aspect est étudié plus en détail par Thépaut et Moll (1990). La non-linéarité de ce modèle de transfert radiatif est le facteur qui empêche une prise en compte aisée des radiances dans l'interpolation optimale (qui est, par nature, linéaire) ; nous avons déjà indiqué que la seule tentative de ce genre était l'analyse Péridot. Mieux, les algorithmes variationnels permettent d'intégrer en un seul concept les différents traitements suivants :

- Restitution d'un profil de température et d'humidité, c'est-à-dire analyse unidimensionnelle (1D), à partir des radiances par inversion variationnelle de l'équation de transfert radiatif.

- Analyse variationnelle tridimensionnelle (3D) utilisant directement les mêmes radiances.

- Assimilation variationnelle quadridimensionnelle (4D) permettant en plus de traiter correctement la dimension temporelle.

Suivant les lignes directrices esquissées ci-dessus, un travail de fond est entrepris dans plusieurs grands centres de prévision numérique, généralement vers la fin des années quatre-vingt. À Reading, en mai 1992, le CEPMMT met en service un système d'inversion interactif pour le Tovs, baptisé $1 \mathrm{D}-\mathrm{VAR}$, suivant la logique de l'analyse 1D. Le profil initial de l'inversion provient de l'ébauche de l'assimilation, c'est-à-dire du modèle (d'où le qualificatif «interactif »). Les centres de Washington (NMC) et de Bracknell (UKMO) effectuent des développements analogues. Le CEPMMT développe aussi, en collaboration avec MétéoFrance, un système d'assimilation variationnelle 3D et 4D (Rabier, 1993). Il rend opérationnelle, en janvier 1995, une analyse variationnelle 3D utilisant directement les radiances Tovs (baptisée 3D-VAR). Développements et expérimentations se poursuivent sur les schémas 4D (baptisés 4D-VAR). 


\section{L'IMPACT ACTUEL DES DONNÉES SATELLITALES} SATELLITALES montrent clairement l'impact positif sur l'hémisphère nord. Plus généralement, si l'on
essaie de porter un jugement d'ensemble sur l'impact des données Tovs sur l'hémi-
sphère nord dans les modèles opérationnels à l'heure actuelle, on peut noter les points
suivants :

- L'amélioration des Satem produits par le Nesdis permet d'obtenir un impact significatif de ces derniers sur les prévisions de l'hémisphère nord, même si l'inversion Nesdis est toujours affectée par les problèmes de fond mentionnés plus haut.

- L'inversion variationnelle interactive (ou 1D-VAR suivant la terminologie du CEPMMT) a aussi permis de progresser dans le domaine de l'utilisation du Tovs.

- Les expériences d'assimilation variationnelle 3D et 4D montrent qu'il existe dans les radiances du Tovs une quantité importante d'information qui n'est pas exploitée dans la plupart des schémas opérationnels, même ceux reposant sur le 1D-VAR. Ainsi, Andersson et al. (1994) montrent l'efficacité d'un schéma 3DVAR pour bien assimiler l'information contenue dans les radiances mesurées dans plusieurs canaux Tovs sans introduire, dans l'analyse, d'autres structures verticales parasites. Ils démontrent aussi comment, dans un schéma 4D-VAR, les canaux vapeur d'eau du Tovs peuvent aider à déterminer le vent en exploitant implicitement le rôle de traceur passif que peuvent jouer certaines structures d'humidité. Une étude récente de Riishojgaard (1995) montre que le canal ozone du Tovs peut être exploité de la même façon.

\section{Impact des données Tovs sur l'hémisphère nord}

Les scores tracés montrent les améliorations apportées sur quatre régions distinctes de l'hémisphère nord par l'utilisation des données Tovs au travers du schéma d'inversion variationnelle 1D-VAR. Les courbes en trait plein (1D-VAR 106) sont les corrélations d'anomalie pour la prévision utilisant les données Tovs (le 106 indiquant la résolution du modèle utilisé en nombres d'ondes). Les courbes en pointillé (OPS 106) sont les corrélations d'anomalie pour la prévision opérationnelle qui sert de référence. Comme cette prévision ne contient pas de données Tovs dans la troposphère de l'hémisphère nord, chaque diagramme montre en fait l'impact des données Tovs 1DVAR troposphériques sur la qualité de la prévision du CEPMMT. Les diagrammes représentent douze cas de prévision en avril et mai 1991.

Notons que les données Tovs ont un impact positif sur les quatre domaines montrés, impact plus fort sur le Pacifique (a) et l'Amérique (b) que sur I'Atlantique (c) et I'Europe (d). Par ailleurs, Kelly et al. (1993) signalent que le bénéfice dû aux Tovs en termes de scores de prévision se trouve plus dans les grandes ondes que dans les ondes synoptiques.
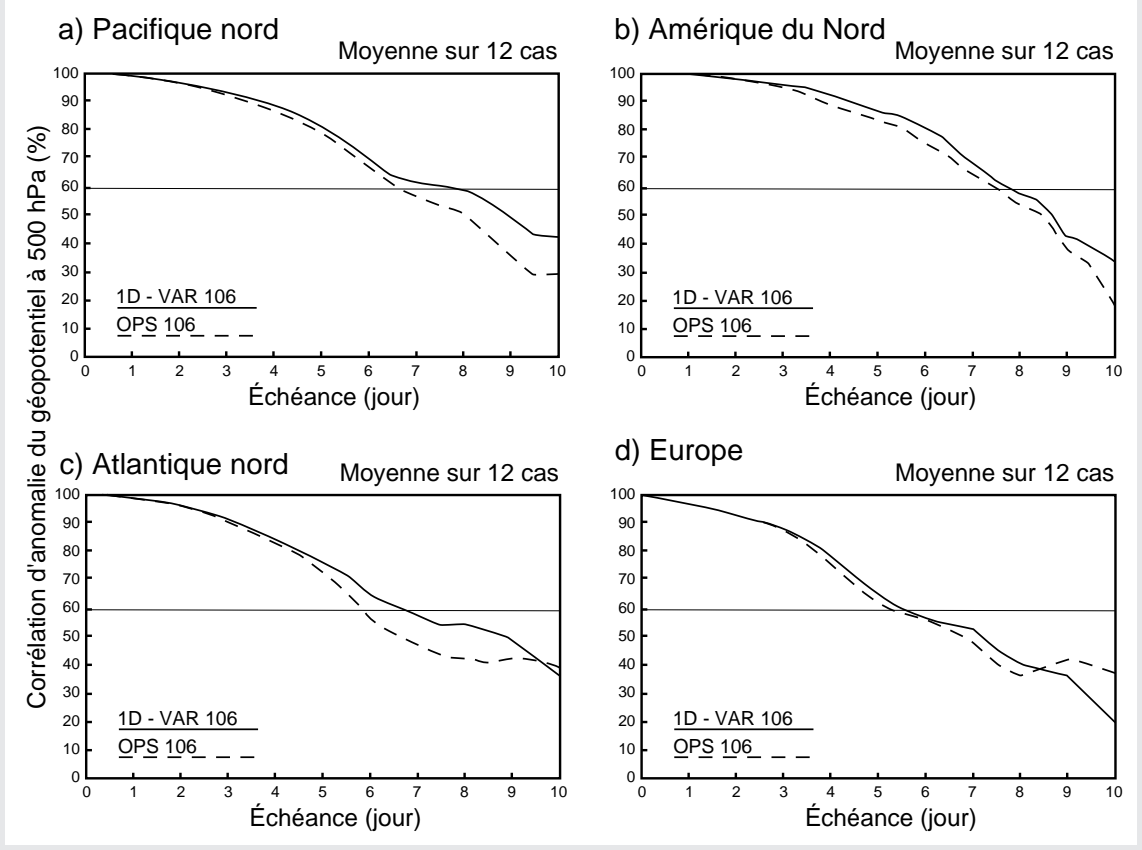

d) Europe

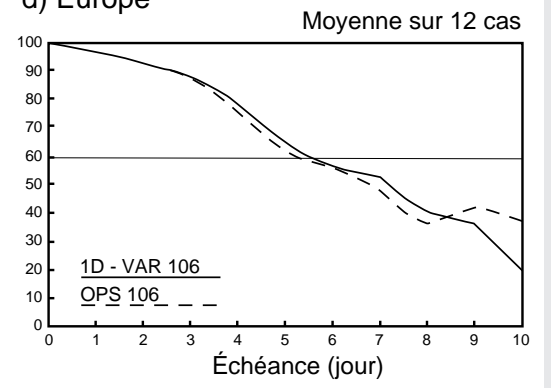


- Il existe dans les données satellitales une grosse quantité d'information sur les nuages. Non seulement, dans l'état actuel des modèles, on ne sait pas bien assimiler cette information, mais encore la plupart des canaux des instruments actuels sont sensibles aux nuages (seuls les canaux micro-ondes y sont relativement insensibles). Bien utiliser ces données suppose d'avoir pu les « décontaminer » de l'influence des nuages, ce qui est une opération délicate en soi et un frein à leur utilisation optimale dans la prévision numérique.

Déduire les vents au moyen d'une assimilation 4D-VAR, en observant des traceurs passifs dans certains canaux, n'est rien d'autre qu'un perfectionnement des méthodes actuelles, employées pour les satellites géostationnaires et consistant à fabriquer des vecteurs vent à partir des déplacements de nuages (ou de certaines structures dans le canal vapeur d'eau) avant de les assimiler. En ce qui concerne les vents, les méthodes d'assimilation ont peu évolué par rapport à ce qui a été fait pour la température et l'humidité. Beaucoup de progrès ont néanmoins été réalisés sur la quantité de vecteurs vent produite (c'est en 1993 que des vents « vapeur d'eau » de Météosat commencent à être diffusés opérationnellement par Darmstadt), ainsi que sur leur qualité. Pour ce qui est de leur impact, nous disposons de beaucoup moins d'études récentes que pour le Tovs. Toutefois, on peut dire que l'impact actuel des Satob sur la prévision numérique n'est pas vraiment différent de ce qui avait été démontré par Kållberg et al. (1982) : fort sous les tropiques, moyen dans l'hémisphère sud et faible dans l'hémisphère nord.

L'impact à méso-échelle des données de satellite est plus difficile à chiffrer et à démontrer. De plus, il s'agit souvent dans ce cas d'un mélange des données provenant des instruments Tovs et AVHRR. Néanmoins, l'étude de Wright et al. (1993), décrivant le système opérationnel britannique Mops (Moisture Observation Pre-processing System), montre qu'à petite échelle l'information satellitale est précieuse pour déterminer les nuages, et que cette information est assimilée avec profit dans les modèles à méso-échelle.

Depuis qu'elles sont utilisées opérationnellement en prévision numérique, les données satellitales ont un rôle déterminant sur l'hémisphère sud et sous les tropiques. Aux latitudes tempérées et polaires, ce sont les sondeurs des satellites défilants qui sont les plus aptes à décrire l'état de l'atmosphère. Aux latitudes équatoriales, ce sont les vents que l'on peut obtenir à partir des satellites géostationnaires. L'intérêt des systèmes d'observation satellitale s'est progressivement renforcé depuis une quinzaine d'années, avec la tendance, dans beaucoup de centres de prévision numérique, à recourir à des modèles opérationnels couvrant tout le globe.

Dans l'hémisphère nord, la question de l'impact des données satellitales est plus subtile. Par rapport à ce que l'on imaginait intuitivement à priori, leur rôle s'est trouvé plus difficile à démontrer et, surtout, l'importance de ce rôle a connu des oscillations au cours de ces quinze dernières années. Si, vers 1980 , personne ne doutait de l'importance des satellites pour la prévision numérique, alors qu'il s'agissait de systèmes d'observation relativement nouveaux à l'époque, leur apport a décliné au cours de la décennie quatre-vingt. Ce n'est que vers le début des années quatre-vingt-dix que, suite à un travail de recherche approfondi, le rôle des satellites en prévision numérique dans l'hémisphère nord apparaît de nouveau indiscutable.

Cette évolution est finalement normale si l'on considère les changements rapides qu'a connus la prévision numérique depuis quinze ans. Dans le même temps, ni les instruments spatiaux ni les méthodes d'assimilation de données n'ont vraiment changé, du moins jusqu'au début des années quatre-vingt-dix. Les efforts pour moderniser les algorithmes d'utilisation des données de satellite commencent à porter aujourd'hui leurs fruits, même si les instruments n'ont pas vraiment changé depuis 1979. Tout porte à croire que le rôle des satellites va encore s'accroître dans les années qui viennent, pour au moins deux raisons :

- Dès 1995, avec l'arrivée de l'instrument Atovs (Advanced Tovs remplaçant le Tovs), une nouvelle génération d'instruments commence à se mettre en place. Elle devrait contribuer à augmenter sensiblement la résolution verticale effective des observations satellitales (surtout en ciel nuageux grâce à de nouveaux 
capteurs micro-ondes). Pour être complet, il faut mentionner la mise en service, en 1991, du satellite ERS 1 équipé en particulier d'un diffusiomètre fournissant des informations sur les vents en surface. ERS 1 est suivi en 1995 d'un satellite ERS 2, et tout indique que ce type de mesures satellitales va être poursuivi en routine. Par rapport aux Satem et aux Satob largement décrits plus haut, on commence donc à assister à une diversification des données satellitales.

- La modernisation des algorithmes d'assimilation de données est loin d'être achevée ; en particulier, le potentiel des méthodes variationnelles (3D ou 4D) est encore presque inexploité aujourd'hui, bien que déjà démontré expérimentalement.

Pour que cet exposé sur l'impact des données satellitales en modélisation soit complet, il faut mentionner le rôle indirect, impossible à quantifier, mais sûrement important, de l'imagerie satellitale. L'utilisation quotidienne de cette imagerie par les prévisionnistes a permis de confronter les résultats de modèles avec la réalité, entraînant du même coup une analyse de leurs faiblesses et des développements judicieux. Dans le même esprit, les satellites ont permis la constitution de bases de données précieuses pour les diagnostics effectués par les modélistes du temps et du climat.

Enfin, la diversification des données satellitales (mentionnée plus haut) entrânera bientôt de nouveaux défis pour ceux qui devront les utiliser dans la prévision numérique et qui ne manqueront pas d'essayer d'en évaluer l'impact. Déjà, les données micro-ondes existantes ne sont pas toutes assimilées, en particulier celles de certains capteurs observant la surface (comme le capteur SSMI embarqué sur certains satellites de défense américains). L'Europe, par l'intermédiaire de l'Agence spatiale européenne (ASE), puis depuis 1986 d'Eumetsat, travaille sur plusieurs programmes ambitieux (Gillet, 1995) :

- Météosat de seconde génération (MSG) ;

- Un projet de satellite à orbite polaire, qui devrait emporter, entre autres, un sondeur infrarouge à très haute résolution, Iasi, financé en grande partie par la France ;

- Un projet de lidar Doppler (c'est le projet Aladin de l'ASE) qui, s'il aboutit, devrait permettre de mesurer le vent sur tout le globe depuis l'espace, et ce avec un très bon échantillonnage de l'atmosphère sur l'horizontale et la verticale.

\section{BIBLIOGRAPHIE}

Andersson E., A. Hollingsworth, G. A. Kelly, P. Lönnberg, J. Pailleux et Z. Zhang, 1991 : Global observing system experiments on operational statistical retrievals of satellite sounding data. Mon. Wea. Rev., 119, 1851-1864.

Andersson E., J. Pailleux, J.-N. Thépaut, J. R. Eyre, A. P. McNally, G. A. Kelly et P. Courtier, 1994 : Use of cloud-cleared radiances in three/four-dimensional variational data assimilation. Quart. J. Roy. Meteor. Soc., 120, 627-653.

Bromley R. A., 1984 : Observing system experiments using the Meteorological Office's 15-level model. Proceedings of the ECMWF seminar on "Data assimilation systems and observing system experiments with particular emphasis on FGGE", Reading, septembre 1984. Disponible auprès du CEPMMT.

Courtier P. and O. Talagrand, 1987 : Variational assimilation of meteorological observations with the adjoint vorticity equation. Part 2: Numerical results. Quart. J. Roy. Meteor. Soc., 113, 1329-1368.

Durand Y., 1985 : The use of satellite data in French high resolution analysis. Proceedings of the ECMWF workshop on "High resolution analysis", Reading, juin 1985. Disponible auprès du CEPMMT.

Eyre J. R., 1989 : Inversion of cloudy satellite radiances by nonlinear optimal estimation. II: Application to TOVS. Quart. J. Roy. Meteor. Soc., 115, 1027-1037.

Gadd A. J., 1993 : Assimilation of TOVS data at the UK Met Office. Proceedings of the ECMWF seminar on "Developments in the use of satellite data in numerical weather prediction", Reading, septembre 1993. Disponible auprès du CEPMMT.

Gallimore R. G. et D. R. Johnson, 1986 : A case study of GWE satellite data impact on GLA assimilation analysis of two ocean cyclones. Mon. Wea. Rev., 114, 2016-2032. 
Gillet M., 1995 : Les nouveaux programmes européens de satellites météorologiques. La Météorologie $8^{\mathrm{e}}$ série, 11, 10-35.

Halem J., E. Kalnay, W. E. Baker et R. Atlas, 1982 : An assessment of the FGGE satellite observing system during SOP-1. Bull. Amer. Meteor. Soc., 63, 407-429.

Kållberg P., S. Uppala, N. Gustafsson et J. Pailleux, 1982 : The impact of cloud track wind data on global analyses and medium range forecasts. ECMWF Technical Report No 34. Disponible auprès du CEPMMT.

Kelly G. A. et J. Pailleux, 1988 : Use of satellite vertical sounder data in the ECMWF analysis system. ECMWF Technical Memorandum No 143. Disponible auprès du CEPMMT

Kelly G. A. et J. Pailleux, 1989 : A study assessing the quality and impact of cloud track winds using the ECMWF analysis and forecast system. Proceedings of the ECMWF/Eumetsat workshop on "The use of satellite data in operational numerical weather prediction: 1989-1993", Reading, mai 1989. Disponible auprès du CEPMMT.

Kelly G. A., E. Andersson, A. Hollingsworth, P. Lönnberg, J. Pailleux et Z. Zhang, 1991 : Quality control of operational physical retrievals of satellite sounding data. Mon. Wea. Rev., 119, 1866-1880.

Kelly G. A., J. Pailleux, F. Rabier et J.-N. Thépaut, 1993 : Observing system experiments made with the ECMWF system. Relevance to the development of some observing systems. WWW Technical Report No 16, WMO/TD No 594. Disponible auprès de l'OMM.

Kidder S. Q. et T. H. Vonder Haar, 1996 : Satellite meteorology. An introduction. Academic Press, San Diego, États-Unis, 466 p.

Le Dimet F.-X. et O. Talagrand, 1986 : Variational algorithms for analysis and assimilation of meteorological observations: theoretical aspects. Tellus, 38A, 97-110.

Lewis J. M. et J. C. Derber, 1985 : The use of adjoint equations to solve a variational adjustment problem with advective constraints. Tellus, 37A, 309-322.

McNally A. P., G. Kelly, J. R. Eyre et E. Andersson, 1993 : Experiments using onedimensional variational analysis of TOVS data at ECMWF. Proceedings of the ECMWF seminar on "Developments in the use of satellite data in numerical weather prediction", Reading, septembre 1993. Disponible auprès du CEPMMT.

Nuret M., 1990 : Production opérationnelle de vecteurs déplacement de nuage à partir de l'imagerie Météosat. La Météorologie 7e série, 31, 7-16.

Pailleux J., 1992 : Use of satellite data in global forecasting. Cospar paper. Adv. Space Res., 12, 7, 253-262.

Pailleux J., 1993 : Assimilation of satellite data using variational techniques. Proceedings of the ECMWF seminar on "Developments in the use of satellite data in numerical weather prediction", Reading, septembre 1993. Disponible auprès du CEPMMT.

Rabier F., 1993 : Assimilation variationnelle de données météorologiques en présence d'instabilité barocline. La Météorologie $8^{\mathrm{e}}$ série, 4, 57-72.

Radford A., 1989 : Monitoring of cloud-motion winds at ECMWF. Proceedings of the ECMWF/Eumetsat workshop on "The use of satellite data in operational numerical weather prediction: 1989-1993", Reading, mai 1989. Disponible auprès du CEPMMT.

Riishojgaard L. P., 1995 : On four-dimensional variational assimilation of ozone data in weather prediction models. Accepté dans Quart. J. Roy. Meteor. Soc.

Smith W. L., H. M. Woolf, C. M. Hayden, D. Q. Wark et L. M. McMillin, 1979 : The TIROS-N Operational Vertical Sounder. Bull. Amer. Meteor. Soc., 60, 1177-1187.

Thépaut J.-N et P. Moll, 1990 : Variational inversion of simulated TOVS radiances using the adjoint technique. Quart. J. Roy. Meteor. Soc., 116, 1425-1428.

Uppala S., A. Hollingsworth, S. Tibaldi et P. Kållberg, 1984 : Results from two recent observing system experiments at ECMWF. Proceedings of the ECMWF seminar on "Data assimilation systems and observing system experiments with particular emphasis on FGGE", Reading, septembre 1984. Disponible auprès du CEPMMT.

Wright B. J., W. H. Hand et B. Macpherson, 1993 : Assimilation of satellite data for mesoscale analysis. Proceedings of the ECMWF seminar on "Developments in the use of satellite data in numerical weather prediction", Reading, septembre 1993. Disponible auprès du CEPMMT. 


\section{Index des sigles et acronymes utilisés}

ASE Agence spatiale européenne.

AVHRR Advanced Very High Resolution Radiometer (imageur des satellites météorologiques défilants).

ATOVS Advanced Tovs (voir Tovs).

CEPMMT Centre européen pour les prévisions météorologiques à moyen terme.

ERS European Remote-sensing Satellite.

EUMETSAT European Meteorological Satellite Agency.

GARP Global Atmospheric Research Program.

GOES Geostationary Operational Environment Satellite (désigne la série de satellites américains géostationnaires).

HIRS High Resolution InfraRed Sounder.

IASI Infrared Atmospheric Sounding Interferometer.

MSG Météosat de seconde génération.

MSU Microwave Sounding Unit.

NOAA National Oceanographic and Atmospheric Administration.

NESDIS National Environment Satellite Data and Information Service.

SATEM Satellite Temperature (nom du code de I'OMM qui sert à transmettre les profils verticaux de température et d'humidité depuis 1977, aux services météorologiques). Surtout utilisé pour désigner ces profils.

SATOB Satellite Observation (nom du code de I'OMM qui sert à transmettre diverses observations de satellites). Surtout utilisé pour désigner les vents déterminés à partir de satellite géostationnaire.

SMT Système mondial de télécommunications.

SSMI Special Sensor Microwave Imager.

SSU Stratospheric Sounding Unit.

TIROS-N Television and InfraRed Observation Satellite (NOAA series).

TOVS Tiros Operational Vertical Sounder.

VTPR Vertical Temperature Profile Radiometer. 